\title{
Reliability Analysis for Bolt-shotcrete Support System in Underground Tunnels
}

\author{
Guangchao Zhang \\ College of Mining and Safety \\ Engineering \\ Shan Dong University of Science \& \\ Technology \\ Qingdao, China \\ zgchao2015@qq.com \\ Bangyou Jiang \\ College of Mining and Safety \\ Engineering \\ Shan Dong University of Science \& \\ Technology \\ Qingdao, China \\ 420395630 @qq.com
}

\author{
Chuanwei Zang* \\ College of Mining and Safety \\ Engineering \\ Shan Dong University of Science \& \\ Technology \\ Qingdao, China \\ chuanweizang@163.com \\ Feng Wang \\ College of Mining and Safety \\ Engineering \\ Shandong University of Science and \\ Technology \\ Qingdao, China \\ 412863764 @qq.com
}

\author{
Zhijie Wen \\ College of Mining and Safety \\ Engineering \\ Shan Dong University of Science \& \\ Technology \\ Qingdao, China \\ sdust0532@gmail.com
}

\begin{abstract}
Reliability analysis of bolt-shotcrete support system is essential for the stability control of the underground tunnels. This paper presented a new analytical method for the reliability analysis of bolt-shotcrete support system. Considering the difference of the stiffness of each support unit, the bolt-shotcrete support structure was divided into two composite structures, and the structural performance function was developed based on the deformation coordination and stress condition of the two composite structures. Due to the nonlinear implicit characteristics of the structural performance function, Rosenblueth method was adopted to solve the function and estimate the failure probability of boltshotcrete system.
\end{abstract}

Keywords--support, reliability analysis, structural performance function, failure probability

\section{INTRODUCTION}

Over the last few decades, more and more Chinese coal mines (especially in the eastern region) are transferring their mining activities to deep coal. Currently, extraction depths of $600 \mathrm{~m}$ or more are becoming more common in China, and at least 20 coal mines have extended their extraction depths to $1000 \mathrm{~m}$ or more below the ground[1]. Due to the high in situ stresses and soft rock characteristics, a deep tunnel generally undergoes large dilation and bulk deformation for a long time after it is excavated, which pose a high risk of various disasters including roof collapse, rib spalling, etc., endangering workers and compromising the functionality of the gateroad[2, 3]. For this reason, various support pattern has been implemented in the deep tunnel, and bolt-shotcrete support is always the primary means for deep tunnel support[4]. However, a large number of field tests have shown that the failure behavior of bolt-shotcrete support structure occurred frequently in deep tunnels. In addition, due to the diversity and complexity of the geological and geotechnical conditions in deep, various influencing factors of bolt-shotcrete support system stability and its bearing capacity has nonnegligible uncertainties[5]. Therefore, reliability analysis of bolt-shotcrete support system is playing an increasingly important in the design of deep gateroad support.
Many attempts have been made to perform a reliability analysis of bolt-shotcrete support system in the past. Zhang et al. developed a limit state equation for bolt- shotcrete support system via Kuper code and obtained corresponding reliability index and partial coefficient[6]. Yang et al. established a structural performance function of a concrete lining, and investigated the variability of the concrete lining deformation and thickness, and materials properties[7]. Bian et al. conducted a reliability analysis for a tunnel boltshotcrete support system using load-structure method[8]. Song et al. analyzed the statistic feature of the initial support performance and developed its reliability index[9]. In these works, a structural performance function was first developed via the simple superposition of the bearing capacity of each support units; then, the structural performance function was solved to obtain the reliability degree of the support system. However, after reviewing these studies, we found two major limitations: (1) Bolt-shotcrete support system is a combination system from the aspect of mechanics. The bearing capacity of this combination system depends on the stiffness of each composite unit, but every composite unit cannot achieve their limit bearing capacity at the same time[10]. Thus, it is unreasonable to evaluate the limit bearing capacity of the support system via the simple superposition of the limit bearing capacity of every composite unit. (2) The forms and complexity of the structural performance function have an important impact on the accuracy and efficiency of the reliability analysis. Due to the complexity of the geological condition of underground tunnels, the structural performance function is generally implicit or non-liner, resulting that it cannot be resolved by the analytic method based on the explicit function. Currently, the most commonly used analytical method are Monte Carlo Simulation(MCS) and Response surface method (RSM). MCS is based on the statistical sampling theory and has the advantage of high accuracy, but it suffers the limitations that it needs a large number of random samples and a longer operation time and a larger workload[11]. RSM is a semi analytical method based on the finite element, but its solution process requires a large amount of repeated iterative with low efficiency[12]. 
Thus, a more effective and precious analytical method is required to provide more realistic results.

In this paper, a structural performance function of boltshotcrete support system, considering the deformation coordination of each support units, was first developed. Then, Rosenblueth method was introduced and used to solve the structural performance function in this study. Finally, taking the case of a shaft station from Xingdong coal mine, China, as an example, the validity of these analytical method was validated. The new analytical method presented in this study can be considered as a viable way for reliability analysis of underground tunnels.

\section{PERFormance FunCtion CONSTRUCTION}

Currently, bolt-shotcrete support is the most effective method of stability control of the underground tunnels. Boltshotcrete support is composed of various support units including shotcrete, reinforcing meshes and bolts, as shown in Fig. 1. For bolt-shotcrete support system, there are several points should keep in mind[13]. (1) The stability of the tunnels not only depends on the support capacity of the shotcrete, reinforcing meshes and bolts, but also depends largely on their interaction and coordination. (2) The interaction between the surrounding rocks and the boltshotcrete support system changes with the increasing of surrounding rocks deformation. This characteristic increases the uncertainty and multiplicity of support effect provided by the bolt-shotcrete system. (3) Local failure of the loading capacity system is common during the service process of the support system. However, the local failure doesn't mean the failure of the whole system[14].

Bolt-shotcrete support system can be simplified to the mechanical model, as shown in Fig.2. The support resistance of the bolt-shotcrete support system is generated by the shotcrete, steel meshes, bolts system and the surrounding rocks in the anchorage zone, respectively. In previous studies, it was assumed that every composite unit (shotcrete, steel meshes, bolts and surrounding rock masses) can achieve their limit bearing capacity at the same time. And thus, the limit bearing capacity of support system can be obtained by (1):

$$
P_{T}=P_{S}+P_{M}+P_{B}+P_{A}
$$

Where $P_{T}$ is the support resistance generated by the whole system, $P_{S}, P_{M}, P_{B}$, and $P_{A}$ are the support resistance generated by shotcrete, reinforcing meshes, bolts and surrounding rock masses in the anchorage zone, respectively.

Based on the theory of Pressure Bearing Arch in boltshotcrete support system, the support resistance supplied by the bolt-shotcrete system $P_{T}$ should not be less than the minimum support resistance $\left(P_{\min }\right)$ required to maintain the stability of the tunnels. Their interrelationship can be expressed as (2):

$$
P_{T}>P_{\min }
$$

According to the theory of the shear-slip[15], the minimum support resistance $P_{\min }$ can be given by (3):

$$
P_{\min }=\gamma\left(R_{\max }-R_{0}\right)
$$

Where $\gamma$ is unit weight of rock mass, $R_{0}$ is the equivalent

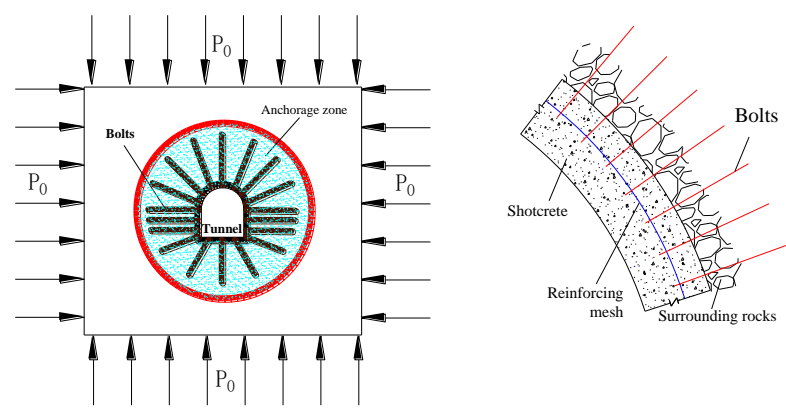

Fig.1 Bolt-shotcrete reinforcement mechanism

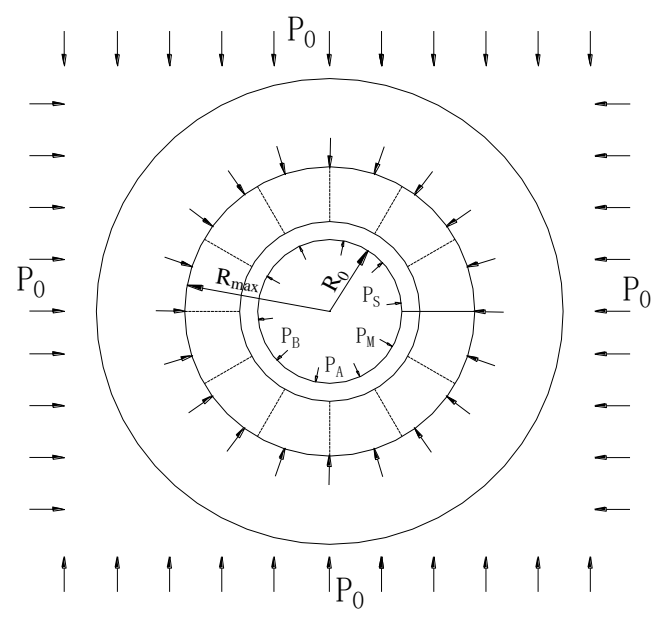

Fig.2 Mechanical model of the bolt-shotcrete support system

radius of the tunnel, and $R_{\max }$ is the range of the fractured zone surrounding the tunnel. According to the theory of elastic-plastic deformation, the $R_{\max }$ can be expressed by (4):

$$
R_{\text {max }}=R_{0}\left[\frac{(1-\sin \varphi)\left(c \cot \varphi+P_{0}\right)}{(1+\sin \varphi)\left(c \cot \varphi+P_{\text {min }}\right)}\right]^{\frac{1-\sin \varphi}{2 \sin \varphi}}
$$

Where $c=c_{0}+\tau_{b} A_{b} / e t, \varphi=\varphi_{0} ; c_{0}$ and $\varphi_{0}$ are the cohesion force and internal friction angle of the surrounding rocks in anchorage zone, $\tau_{b}$ and $A_{b}$ are the shear strength and crosssectional area of the bolts, respectively; $e$ and $t$ are the row and line space of the bolts, respectively; $P_{0}$ is the initial in situ stress.

Substituting (4) into (3), the following equation can be given from (3):

$$
P_{\text {min }}=\gamma R_{0}\left[\frac{(1-\sin \phi)\left(c \cot \phi+P_{0}\right)}{(1+\sin \phi)\left(c \cot \phi+P_{\text {min }}\right)}\right]^{\frac{1-\sin \phi}{2 \sin \phi}}-\gamma R_{0}
$$

In this situation, the structural performance function of support system is given by (6):

$$
Z=P_{T}-P_{\min }
$$

However, in fact, due to the difference of the stiffness of the shotcrete, reinforcing meshes, bolts system and surrounding rocks, they cannot achieve their limit bearing capacity at the same time. Thus, the mentioned construction method of the structural performance function based on the superposition theory is just a simplified method. In light of this limitation, a new construction method was developed in this study, which considers the deformation coordination of each support units. 
Based on the fact that the shotcrete works together with the reinforcing meshes in the field, thus, they can be treated as a composite structure A. The stiffness of this structure is given by (7)[13]:

$$
K_{W}=\frac{E_{C}}{R_{0}} \cdot \frac{R_{0}^{2}-\left(R_{0}-d\right)^{2}}{\left(1+\mu_{c}\right) \cdot\left[\left(1-2 \mu_{c}\right) R_{0}^{2}+2\left(R_{0}-d\right)^{2}\right]}
$$

Where $E_{\mathrm{C}}$ and $\mu_{\mathrm{c}}$ are the elastic modulus and Poisson's ratio of the reinforcing meshes, $d$ is the thickness of the shotcrete. Hence, the relationship between the maximum support resistance of the composite structure $\mathrm{A}$ and its ultimate deformation is given by (8):

$$
P_{1}=K_{W} \cdot u_{1}=P_{S}+P_{M}
$$

Where $p_{1}$ and $u_{1}$ are the maximum support resistance and ultimate deformation of the composite structure A, respectively.

Similarly, the bolts system and rock masses surrounding the tunnel can also be treated as a composite structure B. Its stiffness is given by (9)[13]:

$$
K_{S}=\frac{2 \pi R_{0} F_{W} E_{S}}{\text { Alet }_{C}}
$$

Where $E_{\mathrm{S}}$ is the elastic modulus of the bolts, $l$ is the length of the bolts, $\lambda c$ is the empirical coefficient, $A$ and $F_{\mathrm{W}}$ are the space and area of the transverse steel bars in the reinforcing meshes, respectively. And thus, the maximum support resistance of the composite structure $\mathrm{B}$ is given by (10):

$$
P_{2}=K_{S} \cdot u_{2}=P_{B}+P_{A}
$$

Where $p_{2}$ and $u_{2}$ are the maximum support resistance and ultimate deformation of the composite structure B, respectively.

Due to the difference of the stiffness of the two composite structure $\mathrm{A}$ and $\mathrm{B}$, the total support resistance of the bolt-shotcrete system is not the sum of support resistance generated by the two composite structures. In fact, the total support resistance changes with the deformation of the two composite structure. As follows:

If the composite structure $\mathrm{A}$ reaches its ultimate deformation firstly, namely $u_{1}<u_{2}$, the total support resistance is given by (11):

$$
P_{T}^{(1)}=p_{1}+K_{s} \cdot u_{1}, u=u_{1}<u_{2}
$$

If the composite structure $B$ reaches its ultimate deformation firstly, namely $u_{2}<u_{1}$, the total support resistance is given by (12):

$$
P_{T}^{(2)}=p_{2}+K_{c} \cdot u_{2}, u=u_{2}<u_{1}
$$

If the two composites structure don't reach their ultimate deformation, the total support resistance is given by (13):

$$
P_{T}^{(3)}=K_{c} \cdot u_{2}+K_{s} \cdot u_{1}
$$

Thus, the structural performance function of support system is given by (14):

$$
\left\{\begin{array}{l}
Z=P_{T}^{(1)}-P_{\text {min }}, u=u_{1}<u_{2} \\
Z=P_{T}^{(2)}-P_{\text {min }}, u=u_{2}<u_{1} \\
Z=P_{T}^{(3)}-P_{\text {min }}, u<\left(u_{1}, u_{2}\right)_{\text {min }}
\end{array}\right.
$$

As we can infer from (14) that this structural performance function is an implicit function; in addition, relevant physical and mechanical parameters of rock masses are associated with each other. Therefore, this structural performance function cannot be resolved by common analytical methods.

\section{THE BASIC PRINCIPLE OF ROSENBLUETH METHOD}

In this study, Rosenblueth Method was adopted to solve the structural performance function (14). In this method, it is not necessary to fit the approximate expression of the structural performance function and obtain the probability distribution of the various state variables. What we need to do is to choose two particular points on the limited state surface and determine their mean value and variance; then the first and second moments of the structural performance function are deduced. Finally, the reliability and failure probability of the support system are determined. Compared with other methods, such as MCS and RSM etc., it's a more effective and precious analytical method, and the obtained calculation accuracy can meet the engineering requirement.

Based on the analysis in section 3, the structural performance function can be described as follows:

$$
Z=f\left(x_{1}, x_{2}, x_{3}, \ldots, x_{n}\right)
$$

Where $x_{i}(i=1,2,3, \ldots, \mathrm{n})$ is random variables standing for unit weigh, cohesion force, internal friction angle of rock mass, the thickness of the shotcrete, the line and row space of bolts, etc.

In the case of the distribution function of random variables unknown, what we need to do is to select two value points symmetrically in the interval from $x_{\min }$ to, $x_{\max }$. In general, for a specific variable $x_{i 1}$ with a mean value of $\mu_{i 1}$ and a standard deviation of $\sigma_{i l}$, the following value points can be selected:

$$
\begin{gathered}
x_{i 1}=\mu_{i 1}+\sigma_{i 1} \\
x_{i 1}=\mu_{i 1}-\sigma_{i 1}
\end{gathered}
$$

For $n$ random variables, there are $2 n$ value points and thus resulting $2^{n}$ combinations. Correspondingly, there are $2^{n}$ structural performance function. If we assume that $n$ random variables are independent of each other, and the appeared probability of every combinations is equally, in this case, the mean estimation of $Z$ is given by (18):

$$
\mu_{z}=\frac{1}{2^{n}} \sum_{j=1}^{2^{n}} Z_{j}
$$

If we assume that $n$ random variables are interconnected to each other, and the appeared probability of every combinations is not equivalent, in this case, the probability values $p_{j}$ depends on the correlation coefficient $\rho$ of various random variables. As follows: 


$$
P_{j}=\frac{1}{2^{\mathrm{n}}}\left(1+e_{1} e_{2} \rho_{1,2}+e_{2} e_{3} \rho_{2,3}+\ldots+e_{n-1} e_{n} \rho_{n-1, n}\right)
$$

Where $\rho_{\mathrm{n}-1, \mathrm{n}}$ is the correlation coefficient between the variables $x_{\mathrm{n}-1}$ and $x_{\mathrm{n}}$; if $x_{\mathrm{i}}=x_{\mathrm{i} 1}, e_{i}=1$; if $x_{\mathrm{i}}=x_{\mathrm{i} 2}, e_{i}=-1$. Thus, the mean estimation of $Z$ is given by (20):

$$
\mu_{z}=\sum_{j=1}^{2^{n}} P_{\mathrm{j}} Z_{j}
$$

Based on the estimation of the central moment and original moment, the first and second moment of the random variable $\mathrm{Z}$ are given by (21) and (22):

$$
\begin{gathered}
M_{1}=E(Z)=\mu_{z}=\sum_{j=1}^{2^{n}} P_{j} Z_{j} \\
M_{2}=E\left(Z-u_{z}\right)^{2}=\sigma_{z}^{2}=\sum_{j=1}^{2^{n}} P_{j} Z_{j}-\mu_{z}^{2}
\end{gathered}
$$

Where $M_{1}$ and $M_{2}$ are the first and second moment of the variable moment. (23):

Thus, the corresponding reliability index $\beta$ is given by

$$
\beta=\frac{M_{1}}{\sqrt{M_{2}}}
$$

If the structural performance function is subjected to normal distribution, the failure probability is given by (24):

$$
P_{f}=1-\Phi(\beta)
$$

Where $\Phi(\beta)=\frac{1}{\sqrt{2 \pi}} \int_{-\infty}^{\beta} e^{-\frac{x^{2}}{2}} d x$

\section{CONCLUSION}

Reliability analysis of bolt-shotcrete support system is essential for the stability control of the underground tunnels. The aim of this study is to develop a new analytical method for the reliability analysis of bolt-shotcrete support system. By comparison with previous studies, this work contained the following original aspects. A new construction method of the structural performance function, considering the deformation coordination of each support units, was developed in this study. In this method, the bolt-shotcrete support structure was divided into two composite structures, and the structural performance function was developed based on the deformation coordination and stress condition of the two composite structures. Due to the nonlinear implicit characteristics of the structural performance function, Rosenblueth method was adopted to solve the function and estimate the failure probability of boltshotcrete system.

\section{ACKNOWLEDGMENT}

In this paper, the research was sponsored by Project (2017RCJJ011) supported by the Scientific Research Foundation of Shandong University of Science and Technology for Recruited Talents; Project (01CK03203 and 02CK02302) supported by the Shandong Provincial FirstClass Discipline Fundamental; Project (ZR2018QEE001) supported by the Natural Science Foundation of Shandong Province.

\section{REFERENCES}

[1]. M.C. He. Present situation and prospect of rock mechanics in deep mining engineering. In: Proceedings of the 8th Chinese conference on rock mechanics and engineering, Edited by Chinese Society for Rock Mechanics and Engineering, pp. 88-94, 2004.

[2]. L. Yuan, J.H. Xue and Q.S. Liu. "Surrounding rock stability control theory and support technique in deep rock roadway for coal mine," Journal of the China Coal Society, Vol.36(4), pp.535-543, 2011.

[3]. F.L. He and G.C. Zhang. "Stability analysis and control of deep underground roadways subjected to high horizontal tectonic stress," Journal of China University of Mining \& Technology, Vol.44(3), pp.466-476, 2015. [4]. F.L. He and G.C. Zhang. "Analysis and control of stability of the fractured soft rock surrounding a deep roadway," Rock \& Soil Mechanics, Vol.36(5), pp.1397-1406, 2015.

[5]. Y.H. Su, M.H. Zhao and Y.Y. Zhang. "Numerical method of slope failure probability based on Bishop model. Journal of Central South University, Vol.15(1), pp.100-105, 2008.

[6]. J. Zhang, Y.R. Zhang and D.S. Liu, "Research on object reliability index and partial coefficient of bolt-shotcrete lining for tunnel," Chinese Journal of Rock Mechanics and Engineering, Vol.21(5), pp.671-674, 2002.

[7]. C.Y. Yang, M. Zhang, X.L. Bai, "Displacement-based method for reliability analysis of shotcrete lining of tunnel," Chinese Journal of Rock Mechanics and Engineering, Vol.22(2), pp.266-269, 2003.

[8].G.C. Zhang, S.J. Liang, and Y.L. Tan. "Numerical modeling for longwall pillar design: A case study from a typical longwall, " Journal of Geophysics and Engineering, vol.15, no.1, pp.121-134, 2018.

[9]G.C. Zhang, F.L. He, and Y.L. Lai. "Ground stability of an underground gateroad with $1 \mathrm{~km}$ burial depth : A case study from Xingdong coal mine, China, " Journal of Central South University, vol.25(6), pp.1386-1398, 2018.

[10].G.C. Zhang, Y.L. Tan and S.J. Liang. "Numerical Estimation of Suitable Gob-side Filling Wall Width in a Highly Gassy Longwall Mining Panel, " International Journal of Geomechanics, vol.18, no.8, pp. 04018091, 2018.

[11]. N. Liu. Stochastic Finite Element Method of Reliability and Its Application to Engineering. Beijing: Chinese Water Power Press, 2001.

[12]. Y.X. Song, Y. Liu, Y.Q. Zhu, "Application of response surface method to reliability analysis of monolithic lining of railway tunnel," Chinese Journal of Rock Mechanics \& Engineering, Vol.23(11), pp.18471851, 2004.

[13]. Y.H. Su, M.C. He, Q. Gao, "Application of Rosenblueth method in evaluating stability reliability of anchor-shotcrete net support system for soft-fracture surrounding rock," Chinese Jounal of Geotechnical Engineering, Vol.26(3), pp.378-382, 2004.

[14]. L.V. Rabcewicz. "The new Austrian tunnelling method," Water Power, Vol.16(11), pp.453-456, 1964.

[15]. P. Roussev, "Calculation of the displacements and Pacher's rock pressure curve by the associative law for the fluidity-plastic flow," Tunnelling \& Underground Space Technology, Vol.13(4), pp.441-451, 1998. 